\title{
招請講演
}

\section{Aluminium Intoxication During Chronic Renal Failure}

\author{
D. N. S. Kerr, MSc., FRCP (L), FRCP (E) \\ University of Newcastle upon Tyne, NE1 4LP, England.
}

The syndromes caused by aluminium intoxication in patients with renal failure will be discussed in the light of our experience and other reports in the world literature.

Source of aluminium

A modest rise in serum aluminium occurs in chronic renal failure, before the institution of dialysis or phosphate binder therapy, typically to about twice normal. This presumably reflects retention of aluminium in food. A more striking rise occurs when aluminium hydroxide is given orally, serum levels occasionally exceeding $100 \mu \mathrm{g} / l$ ( 4 $\mu \mathrm{mol} / l$. There are a handful of cases in the whole world literature, in which aluminium intoxication from this source has caused serious disease or death. Children are more susceptible than adults. In general, the advantages of phosphate binders far outweigh their risks.

The usual cause of aluminium poisoning has been absorption from dialysate during haemodialysis. Water used to make dialysis fluid is the common source of the aluminium. An aluminium concentration greater than $10 \mu \mathrm{g} /$ $l(0.4 \mu \mathrm{mol} / l)$ in water is unacceptable and calls for water treatment. The effect of reverse osmosis and of deionisation varies with the individual water supply; the treatment plant should be tailor-made to the local supply and treated water should be monitored regularly. Accurate control of the $\mathrm{pH}$ of dialysis flyid at 7.4 minimises absorption of any aluminium which escapes removal or comes from the concentrate.

The best available CAPD fluid contains about $17 \mu \mathrm{g} / l(0.6 \mu \mathrm{mol} / l)$ and results in a modest rise in serum aluminium during the first year of treatment. One outbreak of acute aluminium poisoing has occurred in Europe from accidental contamination of one supplier's fluid.

\section{Clinical syndromes}

The well described syndrome of dialysis encephalopathy will be reviewed. Less well known manifestations are bone disease and anaemia. The osteodystrophy is a severe osteomalacia, with little evidence of hyperparathyroidism, causing bone pain, myopathy and fracture. It does not respond to vitamin D analogues. Hypercalcaemia is readily precipitated by vitamin $\mathrm{D}$ or calcium. Bone biopsy shows aluminium concentrated at the ossification front.

Treatment

Aluminium intoxication is easier to prevent than to treat. However, rigorous exclusion of aluminium from dialysis fluid and medications has produced gratifying improvement in early encephalopathy and recovery from the microcytic anaemia. Aluminium excretion can be accelerated by administering desferioxamine. Bone disease improves symptomatically but the radiological and pathological changes take years to repair, even after successful renal transplanatation. 